\title{
Multiresistant Neisseria gonorrhoeae: a new threat in second decade of the XXI century
}

\author{
Beata Młynarczyk-Bonikowska ${ }^{1} \cdot$ Anna Majewska $^{2}$ - $\cdot$ Magdalena Malejczyk ${ }^{1} \cdot$ Grażyna Młynarczyk $^{2}$. \\ Sławomir Majewski ${ }^{3}$
}

Received: 25 November 2018 / Accepted: 26 November 2019 / Published online: 4 December 2019

(c) The Author(s) 2019, corrected publication [2020]

\begin{abstract}
Neisseria gonorrhoeae is an etiologic agent of gonorrhoea, one of the most common sexually transmitted diseases caused by bacteria. For many years, infections caused by $N$. gonorrhoeae were considered to be relatively easy to treat; however, resistance has emerged successively to all therapeutic agents used in treatment of the disease, e.g., penicillin, ciprofloxacin or azithromycin. Currently, the global problem is the emergence and a threat of spread of $N$. gonorrhoeae strains resistant to extended-spectrum cephalosporins (ESC), such as injectable ceftriaxone and oral-used cefixime. Especially, dangerous are multi-resistant strains resistant simultaneously to ESC and azithromycin. Three strains with high-level resistance to azithromycin and resistant to ESC were first time isolated in 2018. Moreover, in 2018, the first ESBL was described in $N$. gonorrhoeae and that makes the threat of appearing the ESBL mechanism of resistance in $N$. gonorrhoeae more real, even though the strain was sensitive to ceftriaxone. Molecular typing revealed that variants resistant to ESC occurred also among strains belonging to epidemic clonal complex CC1 (genogroup G1407) distinguished in NG-MAST typing system. The G1407 genogroup, in particular the ST1407 sequence type, is currently dominant in most European countries. The presence of different mechanisms of drug resistance significantly affects clinical practice and force changes in treatment regimens and introduction of new drugs.
\end{abstract}

Keywords Epidemic clones NG-MAST $\cdot$ Gonorrhoea $\cdot$ Mechanisms of antibiotic resistance $\cdot$ Multiresistant Neisseria gonorrhoeae

\section{Introduction}

Gonorrhoea is one of the most common sexually transmitted bacterial diseases in the world [1]. Several bacterial or viral diseases can be transmitted simultaneously that may need the adequate diagnostics and therapy [2,3]. Gonorrhoea

Edited by Volkhard A. J. Kempf.

Anna Majewska

anna.majewska@wum.edu.pl

1 Department of Diagnostics of Sexually Transmitted Diseases, Medical University of Warsaw, 82a Koszykowa Str, 02-008 Warsaw, Poland

2 Department of Medical Microbiology, Medical University of Warsaw, 5 Chalubinskiego Str, 02-004 Warsaw, Poland

3 Department of Dermatology and Venereology, Medical University of Warsaw, 82a Koszykowa Str, 02-008 Warsaw, Poland diagnostics can be carried out using both culture and molecular methods, but bacterial culture is necessary to determine drug susceptibility $[4,5]$. According to the WHO data, the number of gonorrhoea patients amounted to over 78 million people per year [6], and this value may be greatly underestimated considering a relative ease of treatment and incomplete registration of infections in many countries. Single doses of third-generation cephalosporins, such as ceftriaxone or cefixime are used in the treatment of gonorrhoea in the majority of countries in the world. The emergence of $N$. gonorrhoeae strains resistant to ceftriaxone and cefixime, and additionally resistant to penicillin, fluoroquinolones, tetracyclines and azithromycin, on different continents in 2007-2018 aroused great concern around the world [7-10]. This was the reason for the search for new drugs or alternative therapies active against $N$. gonorrhoeae. An example of new inhibitors of bacterial topoisomerase, that could be used in the treatment of gonorrhoea are fluoroquinolones: delafloxacin, sitafloxacin or two drugs in clinical investigations, 
zoliflodacin and gepotidacin. Examples of protein synthesis inhibitors are new drugs that entered clinical trials: lefamulin and solithromycin and drugs only in preclinical tests: apramycin (aminoglycoside) and aminomethyl spectinomycins [11-18]. Another candidate for gonorrhoea treatment with a new mechanism of action, a small antimicrobial molecule SMT-571, demonstrated in vitro high activity against all investigated strains of $N$. gonorrhoeae [19]. Aside from usage of old and new drugs alone or in combinations, alternative therapies with probiotics or bacteriophages are taken into consideration. Antigenic variability makes the development of gonococcal vaccine difficult. Only a few vaccines entered clinical trials and all of them were unsuccessful. Recently, the animal trials with LOS-derived OS epitope (2C7) gave the promising results. Another interesting fact is that persons vaccinated against serogroup B meningococci are partially immune against gonorrhoea [20, 21] In 2017, gonococci were included into the WHO list of 12 pathogens, whose drug resistance is a global health threat, and which most urgently require the creation of new antibiotics [22].

\section{Spreading of multi-drug resistant variants of $\boldsymbol{N}$. gonorrhoeae and their importance}

The problem of bacterial drug resistance can be considered at various levels. It is particularly important to understand that the evolution of resistance mechanisms in bacteria is inextricably associated with the epidemiology of infections with a given species. The emergence of a new resistance mechanism is much more important if it occurs in specific epidemic or pandemic clones, which for various reasons are characterized by increased infectiveness and/or pathogenicity and are, therefore, responsible for a majority of infections with a particular species. The representatives of the CC1 clonal complex NG-MAST (G1407 genogroup NG-MAST) [23] according to the molecular typing system NG-MAST (Neisseria gonorrhoeae multi-antigen sequence typing), which is most frequently used in the epidemiological studies of $N$. gonorrhoeae (MLST, NG-STAR, MLVA and other systems are also used), seem to play the dominant role in N. gonorrhoeae. The G1407 genogroup, in particular the ST1407 sequence type, is currently dominant in most European countries. This genogroup includes about 330 sequence types, also including ST3128, ST3158, ST4120, ST5332, which dominate or have a significant share in individual countries [24, 25]. The interest in resistance mechanisms remains closely related to clinical practice. The emerging resistance mechanisms force the introduction of new drugs and change of treatment regimens. The timetable of appearance of the resistance in $N$. gonorrhoeae to particular drugs is shown in the Table 1 [7, 15, 26-30]. The arising, presently still rare in this species, resistance to third-generation cephalosporins is of particular importance for $N$. gonorrhoeae. However, it was observed that the mechanisms of resistance to drugs formerly used in gonorrhoea treatment, i.e., plasmid-coded, once very popular (such as the resistance to penicillin related to beta-lactamase production or TetM proteins related to tetracycline resistance) do not occur at all or are very rare in the currently dominating sequence
Table 1 The year of introduction of drug and first report of resistance

\begin{tabular}{|c|c|c|c|}
\hline \multirow[t]{2}{*}{ Drug } & \multirow{2}{*}{$\begin{array}{l}\text { Intro- } \\
\text { duction } \\
\text { (year) }\end{array}$} & \multicolumn{2}{|l|}{ Resistance } \\
\hline & & Year and country & Mechanism \\
\hline Sulfonamides & 1930 & 1945 & Probably chromosomal mutation \\
\hline Penicillin & 1943 & $\begin{array}{l}1945 \\
1980 \text { Canada } \\
1976 \text { England, USA }\end{array}$ & $\begin{array}{l}\text { Probably chromosomal mutation } \\
\text { Chromosomal mutation } \\
\text { Plasmid encoding beta-lactamase (PPNG strains) }\end{array}$ \\
\hline Tetracyclines & 1949 & $\begin{array}{l}1969 \text { USA } \\
1985 \text { USA }\end{array}$ & $\begin{array}{l}\text { Probably chromosomal mutation } \\
\text { Plasmid encoding TetM }\end{array}$ \\
\hline Spectinomycin & 1967 & $\begin{array}{l}1973 \text { England } \\
1981 \text { England }\end{array}$ & $\begin{array}{l}\text { Chromosomal mutation } \\
\text { Spectinomycin resistant PPNG }\end{array}$ \\
\hline Ciprofloxacin & 1980 & 1990 USA & Chromosomal mutation \\
\hline Azithromycin & 1988 & $\begin{array}{l}1991 \text { USA } \\
1997 \text { USA }\end{array}$ & $\begin{array}{l}\text { ermF } \\
\text { Chromosomal mutation 23S rRNA or } m t r\end{array}$ \\
\hline $\begin{array}{l}\text { CME } \\
\text { Ceftriaxone } \\
\text { Cefixime }\end{array}$ & 1989 & $\begin{array}{l}2007 \text { China } \\
\text { 2009 Japan } \\
\text { 2010 France } \\
\text { 2018 England } \\
\text { Australia }\end{array}$ & $\begin{array}{l}\text { Chromosomal mutation penA (ceftriaxone } \\
\text { MIC }=0.5 \mathrm{mg} / \mathrm{L} \text { ) } \\
\text { Chromosomal mutation penA (ceftriaxone } \\
\text { MIC }=2.0 \mathrm{mg} / \mathrm{L} \text { ) } \\
\text { Chromosomal mutation penA (ceftriaxone } \\
\text { MIC }=1.0 \mathrm{mg} / \mathrm{L} \text { ) } \\
\text { Chromosomal mutation penA and } 23 \mathrm{~S} \text { rRNA } \\
\text { (ceftriaxone } \mathrm{MIC}=0.5 \mathrm{mg} / \mathrm{L} \text { and azithromycin } \\
\text { MIC }>256 \mathrm{mg} / \mathrm{L} \text { ) }\end{array}$ \\
\hline
\end{tabular}


types in Europe. In these bacteria, mechanisms involving the synthesis of specific plasmid-coded proteins have been replaced by other mechanisms, conditioned by mutations of chromosomal genes. The transfer of beta-lactamase plasmid to N. gonorrhoeae belonging to genogroup ST1407 is possible, but the bacteria cannot maintain the plasmid for longer time [31]. This indicates a tendency of removing the energy-intensive ballast by bacteria in the absence of selective pressure in the environment. The level of resistance to a specific antibiotic, measured by the MIC value, is much lower in these cases. For this reason, it is especially important to adopt appropriate criteria for recognizing a bacterial strain as resistant or susceptible to a particular drug. Generally accepted criteria are determined annually by institutions appointed for this purpose, such as EUCAST (The European Committee on Antimicrobial Susceptibility Testing), obligatory in many European countries, and CLSI (Clinical and Laboratory Standards Institute), obligatory in the USA and many non-European countries. These criteria may differ from each other and may change in successive years. In European countries, accuracy of antimicrobial susceptibility tests is checked by appropriate and regular quality assurance procedures [32]. Current EUCAST and CLSI (March 2019) breakpoints are presented in Table 2 [33, 34]. Therefore, when specifying the percentage of bacteria susceptible to a given antibiotic, it is necessary to provide the accepted susceptibility criteria, or to calculate the $\mathrm{MIC}_{50}$ and $\mathrm{MIC}_{90}$ values. The term "resistance" is abused in relation to certain mechanisms. In N. gonorrhoeae, for example, the mechanism related to the overproduction of MtrCDE, MacAB or NorM membrane pumps, unless it interacts with another mechanism, does not cause resistance, but only decreases the susceptibility (increase in the MIC value of the drug) and the strain is classified as susceptible.

Table 2 MIC breakpoints for Neisseria gonorrhoeae

\begin{tabular}{llllll}
\hline Drug & \multicolumn{3}{l}{ MIC breakpoints (mg/L) } \\
\cline { 2 - 3 } & \multicolumn{2}{l}{ EUCAST 2019 } & & \multicolumn{2}{l}{ CLSI 2019 } \\
\cline { 2 - 3 } \cline { 5 - 6 } & $S \leq$ & & $R \leq$ & $R \geq$ \\
\hline Benzylpenicillin & 0.06 & 1.0 & & 0.06 & 2.0 \\
Cefixime & 0.125 & 0.125 & & 0.25 & - \\
Cefotaxime & 0.125 & 0.125 & & 0.25 & - \\
Ceftriaxone & 0.125 & 0.125 & & 0.25 & - \\
Ciprofloxacin & 0.03 & 0.06 & & 0.06 & 1.0 \\
Ofloxacin & 0.125 & 0.25 & & 0.25 & 2 \\
Azithromycin* & 1.0 & 1.0 & & 1.0 & - \\
Tetracycline & 0.5 & 1.0 & 0.25 & 2.0 \\
Spectinomycin & 64 & 64 & 32.0 & 128.0 \\
\hline
\end{tabular}

*Azithromycin is always used in conjunction with another effective agent

\section{Acquisition of drug resistance by $\mathbf{N}$. gonorrhoeae}

The mechanism of acquiring resistance is another area, in which the problem of resistance to antibacterial drugs can be considered. The acquisition of a new mechanism of resistance by epidemic clones in the majority of both Gram-positive and Gram-negative bacteria occurs as a result of external uptake, usually by conjugation, of mobile genomic elements (plasmid, transposon, gene cassettes integrated into integron, genomic resistance islands, etc.). The transmission of chromosomal genes with mutations that occurred in the donor through transformation in natural conditions has been described only in the genus Neisseria and to a much smaller extent in Streptococcus. Resistance or reduced susceptibility to antibacterial drugs in currently dominant $N$. gonorrhoeae clones is most often associated with mutational changes in chromosomal genes. One or more mutations (so-called non-mosaic changes involving one or several amino acids in the PBP2 protein) or a combination of multiple mutations, the effect of which is a change from 21 to more than 60 amino acids in the coded protein (so-called mosaic or semimosaic changes), are required for the emergence of resistance in some genes (e.g., penA). The unique ability among bacteria of the genus Neisseria to uptake chromosomal DNA with a high frequency through transformation from other genera of Neisseria allows to accumulate many mutations in chromosomal genes. Most bacteria need to acquire the state of competence to uptake DNA through transformation. Bacteria from the genus Neisseria remain constantly in the state of competence, and moreover, Neisseria chromosomal DNA has specific sequences, known as 10 bp DUS (DNA uptake sequence), and GCCGTCTGAA sequences that act as a generic-specific DNA marker. There are 1965 copies of the DUS sequence in a $2.15-\mathrm{Mb}$ chromosome, which means that they occur on average every $1096 \mathrm{bp}$ [35]. DUS sequences enable bacteria, which uptake DNA by means of transformation, to determine if it is derived from the genus Neisseria. Extrachromosomal DNA (plasmids) does not contain DUS markers and is transformed at a much lower frequency. Plasmid transmission is most often carried out by conjugation (conjugative plasmids, non-conjugative plasmids mobilized by conjugative elements). The most important is the uptake of altered genes or their fragments, determining conditioning resistance to drugs, through $N$. gonorrhoeae or $N$. meningitidis epidemic or pandemic clones. Not every mutation that reduces antibiotic susceptibility, taken up through transformation, even by an epidemic clone, guarantees evolutionary success. It should be noted that the mutation, despite the fact that it determines resistance (which guarantees 
the clone an advantage in the case of selective pressure, e.g., during treatment), may also have a negative impact on the physiological functions of the altered protein and related proteins, e.g., within various regulatory systems. Reduced or altered metabolic activity of some proteins may affect infectivity, colonization capacity, metabolic rate, mechanisms that protect the cell against the immune system (quantitatively or qualitatively), which may be a significant obstacle in gaining a quantitative advantage in confrontation with clones without metabolic defects.

\section{Resistance to beta-lactams}

Beta-lactam antibiotics have a bactericidal effect against $N$. gonorrhoeae, as a result of inactivation of the transpeptidase domain in PBP2 and PBP1 proteins involved in peptidoglycan synthesis. Penicillin action is based on acylation of serine at position 310 in N. gonorrhoeae PBP2 [36]. The penicillin MIC for strains with chromosomal resistance to penicillin is most often between 2.0 and $4.0 \mathrm{mg} / \mathrm{L}$, and from 4.0 to $32.0 \mathrm{mg} / \mathrm{L}$ for beta-lactamase-producing strains. Strains with MIC $\geq 0.25 \mathrm{mg} / \mathrm{L}$ are considered resistant to ceftriaxone and cefixime according to the European criteria. CLSI did not specify criteria for resistant strains (Table 2).

\section{Chromosomal resistance to penicillin and oxyimino-cephalosporins}

The first $N$. gonorrhoeae strain with chromosomal resistance to penicillin was described in the USA in North Carolina in 1983 [37]. Currently, the global problem is the threat of emergence and spread of $N$. gonorrhoeae strains resistant to extended-spectrum cephalosporins (ESC), such as ceftriaxone and cefixime. However, only a few strains with ESC resistance have been described so far. The first such strain, HO41 resistant to cefixime $(\mathrm{MIC}=4 \mathrm{mg} / \mathrm{L})$ and ceftriaxone (MIC $=2 \mathrm{mg} / \mathrm{L}$ ), was isolated in 2009, in Kyoto, Japan [29, 38]. No further ESC-resistant $N$. gonorrhoeae strains were found in the Kyoto and Osaka regions in 2010-2012, indicating that HO41 was not an epidemic clone and did not spread in the population [39]. However, some other resistant strains appeared in different countries. The $N$. gonorrhoeae strains with ceftriaxone MIC $\geq 0.5 \mathrm{mg} / \mathrm{L}$ are presented in Table 3 $[7,8,29,30,40-51]$.

Table $3 N$. gonorrhoeae strains with ceftriaxone MIC $\geq 0.5 \mathrm{mg} / \mathrm{L}$

\begin{tabular}{|c|c|c|c|c|c|c|c|c|}
\hline Country & Year & Strain & ST NG-MAST & $\begin{array}{l}\text { Genogroup } \\
\text { NG-MAST }\end{array}$ & ST MLST & ST NG-STAR & PenA & $\begin{array}{l}\text { CTX } \\
\text { MIC } \\
\mathrm{mg} / \mathrm{L}\end{array}$ \\
\hline Ireland & 2018 & IR72 & 17,842 & G564 & 1903 & 1133 & 60.001 & 0.5 \\
\hline Australia & 2018 & A2543* & 16,848 & G1866 & 12,039 & 996 & 64.001 & 0.5 \\
\hline England & 2018 & G97687* & 16,848 & G1866 & 12,039 & 996 & 64.001 & 0.5 \\
\hline England & 2018 & G7944* & 16,848 & G1866 & 12,039 & 996 & 64.001 & 0.5 \\
\hline France & 2017 & F90 & 3435 & G564 & 1903 & 133 & 60.001 & 0.5 \\
\hline Denmark & 2017 & GK124 & 1614 & G5267 & 1903 & ND & ND & 0.5 \\
\hline Canada & 2017 & 47,707 & 1614 & G5267 & 1903 & 233 & 60.001 & 1.0 \\
\hline Australia & 2017 & A7536 & 15,925 & G11110 & 1903 & 233 & 60.001 & 0.5 \\
\hline Australia & 2017 & A7846 & 1614 & G5267 & 1903 & 233 & 60.001 & 0.5 \\
\hline Japan & 2015 & FC428 & 3435 & G564 & 1903 & 233 & 60.001 & 0.5 \\
\hline Japan & 2015 & FC460 & 3435 & G564 & 1903 & 233 & 60.001 & 0.5 \\
\hline Japan & 2014 & GU140106 & 6543 & G11018 & 7363 & ND & ND & 0.5 \\
\hline Argentina & 2014 & CCETS-7069 & 13,064 & $\mathrm{G} 21$ & 13,637 & 139 & IX & 0.5 \\
\hline Australia & 2013 & A8806; WHO-Z & 4015 & G11018 & 7363 & 227 & 64.001 & 0.5 \\
\hline China & $2012 / 13$ & GD4 & 10,208 & G5062 & ND & ND & II & 0.5 \\
\hline China & $2012 / 13$ & GX1 & 11,129 & ND & ND & ND & XXI & 0.5 \\
\hline China & $2012 / 13$ & HN9 & 5913 & G1791 & ND & ND & XXI & 0.5 \\
\hline Spain & 2012 & F89; WHO-Y & 1407 & G1407 & 1901 & 16 & 42.001 & 1.0 \\
\hline France & 2010 & F89; WHO-Y & 1407 & G1407 & 1901 & 16 & 42.001 & 1.0 \\
\hline Japan & 2009 & HO41;WHO-X & 4220 & G4019 & 7363 & 226 & 37.001 & 2.0 \\
\hline China & 2007 & ND & 2288 & G1791 & ND & ND & XVII & 0.5 \\
\hline
\end{tabular}

$N D$ not done, $C T X$ ceftriaxone

*Azithromycin resistance, MIC $>256 \mathrm{mg} / \mathrm{L}$ 
In 2018, three strains resistant to ceftriaxone and azithromycin $(\mathrm{MIC}=256 \mathrm{mg} / \mathrm{L})$ were described in Great Britain and Australia (two strains) [44, 46, 52]. In 2011-2015, 26 strains resistant to ceftriaxone (MIC $>0.125 \mathrm{mg} / \mathrm{L}$ ) were detected as part of the Euro-Gasp program. These strains occurred in Germany $(n=10)$, Spain $(n=6)$, Greece $(n=4)$, Austria $(n=3)$ and one strain in Norway, Ireland and Slovenia [53]. Fifteen strains resistant to ceftriaxone were described as a result of retrospective studies of strains isolated in China in 2007 [7]. Subsequent studies of N. gonorrhoeae strains allowed characterization of another 29 strains resistant to ceftriaxone [8].

The reported increased in $N$. gonorrhoeae MICs of the ESC currently used in gonorrhoea treatment, ceftriaxone and cefixime and of penicillin are probably the effect of mutations in the chromosomal region of the penA gene (encoding the transpeptidase domain of the $\mathrm{PBP} 2$ protein), porB $1 b$ gene (encoding the porin B subunit), ponA gene (encoding the PBP1 protein) and overexpression of MtrCDE membrane pump proteins. Mutational changes in the penA gene play the most important role in the emergence of chromosomal resistance or reduced susceptibility to ESC. The mutated penA gene is probably acquired by transformation from commensal bacteria Neisseria cinerea [54]. The resulting changes in the amino acid pattern of the PBP2 protein (most often substitutions and single deletions and insertions of amino acids) are referred to as PBP2 mosaic, semimosaic or non-mosaic patterns. Sixty-four patterns of the PBP2 protein are described (85-576 amino acid region). Amino acid sequences of the PB2-2A protein of the $N$. gonorrhoeae M32091 strain were assumed as the wild type (pattern 0). Patterns I-IX, XI-XXII, XXIV, XXXIII, 40, 41, 43-46, 48-50, 54, 56, 57 and 61, are defined as non-mosaic (number of amino acid changes from 1 to 13). Patterns XXIII, XXXV and 39 were defined as semimosaic (number of amino acid changes from 22 to 31 ). The remaining ones are considered mosaic patterns (number of amino acid changes from 33 to 61) [23]. The described PBP2 patterns determine different MIC values of ESC and penicillin $[23,55,56]$. The increase in ESC MIC (reduced susceptibility or resistance to ESC, ceftriaxone MIC of $0.125-0.25 \mathrm{mg} / \mathrm{L}$ ) is characteristic for some $N$. gonorrhoeae carrying PBP2 not only with changes, referred to as mosaic patterns (amino acid substitutions probably conditioning resistance or reduced susceptibility are: A311V, I312M, V316T or P, T484S, A502P, G546S) occurring more frequently in European countries, but also with non-mosaic patterns containing substitutions: A502V, A502T, G543S, P552S in PBP2 and usually a D346 insertion (more often in Asian countries) [5, 7, 8, 57, 58]. The HO41 and F89 strains, showing high resistance to ESC, have the PBP2 37 (former name: $C$ ) and 42 mosaic patterns (former name: CI). PBP2 patterns, according to Demczuk et al. [23], including amino acid substitutions and insertions determining reduced susceptibility or resistance to ESC are presented in Table 4 [7, 8, 23, 29, 30, 47, 48].

In addition to mutations in the PBP2 encoding genes, the increase in penicillin MIC may also be conditioned by mutations in the penC gene (alternative gene name: pilQ), encoding PilQ secretin [59]. However, analyses of strains isolated in South Korea [60] showed that N. gonorrhoeae strains exhibiting the same changes in the genes coding for PBP2, MtrR, PBP1 and porin B differed significantly in the MIC of ceftriaxone, cefixime, cefpodoxime and penicillin $\mathrm{G}$. This indicates that these gene alterations do not fully explain the reasons for beta-lactam MICs.

The highest percentage of cefixime-resistant $N$. gonorrhoeae among European countries in 2016 was recorded in Croatia (11.1\%), Luxembourg (10\%), Belgium (8.1\%), Germany (6.4\%), Poland (5.2\%), Austria (4.2\%) and Slovakia (3.6\%) [61]. In some European countries, i.e., in Poland, the strains were isolated first time in 2016; while, an increase in the number of strains with reduced susceptibility to ceftriaxone ( $\mathrm{MIC}=0.125 \mathrm{mg} / \mathrm{L})$ and decrease in penicillin-susceptible strains were observed in previous years [62, 63]. No cases of resistance to ceftriaxone were detected in 2016 in Europe [61].

\section{Plasmid mediated resistance to penicillins}

The first $N$. gonorrhoeae producing beta-lactamase was described in Great Britain in 1976 [64]. Since then, until 2018, nine different beta-lactamases were isolated from $N$. gonorrhoeae, all from the TEM group. Beta-lactamases are encoded by a family of non-conjugative penicillin plasmids that differ in deletions, insertions or duplications. These changes are related to the described earlier Asia plasmid (4.4 MDa, 7426 bp, pJD4 prototype). Plasmids described so far include: Africa (3.2 MDa, 5599 bp, 1880-3708 deletion, pJD5 prototype), Toronto/Rio (3.05 MDa, 5154 bp, 3802-6075 deletion, pJD7 and pGO4717 prototypes), Nimes (3.8 MDa, 6798 bp, 1880-3708 deletion and IS5 insertion 1200 bp between nucleotides 604-605, pGF1 prototype), New Zealand (8.5 MDa, 9309 bp, TR duplication tandem repeat, 1883 bp from nucleotide 593), Johannesburg (4865 bp, two deletions: 1928-4487 and 6236, pEM1 prototype), Australia (3269 bp, two deletions: 1928-4487 and 3795-6066 and T7424C transitions) [65-69].

Beta-lactamases described in N. gonorrhoeae are: TEM1 , occurring in all types of penicillinase plasmids previously described in N. gonorrhoeae, TEM-135 (M182T substitution) most commonly found in the Toronto/Rio plasmids, TEM-220 (M182T and A185T substitutions) described in the Toronto/Rio plasmids, TEM (E110K substitution) described in the Africa plasmid, TEM (G228S substitution) described in the Africa plasmid and TEM (Q269K 
Table 4 Changes of the amino acids in the N.gonorrhoeae PBP2 protein transpeptidase domain including sites determining reduced sensitivity or resistance to oxyimino-cephalosporins

\begin{tabular}{|c|c|c|c|c|c|c|c|c|c|c|c|c|c|c|c|c|c|c|c|}
\hline \multirow[t]{5}{*}{ No PBP2 } & \multirow[t]{5}{*}{$\mathrm{NC} / \mathrm{TC}$} & \multicolumn{9}{|c|}{ Selected changes (aa) } & \multirow[t]{5}{*}{ No PBP2 } & \multirow[t]{5}{*}{$\mathrm{NC} / \mathrm{TC}$} & \multicolumn{6}{|c|}{ Selected changes (aa) } & \\
\hline & & A & I & V & $\square$ & $\mathrm{T}$ & A & G & G & $P$ & & & A & I & V & ᄃ & & & $\mathrm{G}$ \\
\hline & & 3 & 3 & 3 & 3 & 4 & 5 & 5 & 5 & 5 & & & 3 & 3 & 3 & 3 & & & 5 \\
\hline & & 1 & 1 & 1 & 4 & 8 & 0 & 4 & 4 & 5 & & & 1 & 1 & 1 & 4 & & & 4 \\
\hline & & 1 & 2 & 6 & 6 & 4 & 2 & 3 & 6 & 2 & & & 1 & 2 & 6 & 6 & & & 3 \\
\hline I & $1 / \mathrm{n}$ & & & & $\mathrm{D}$ & & & & & & XXXIII & $7 / \mathrm{n}$ & & - & - & D & & & $S$ \\
\hline & $5 / \mathrm{n}$ & & 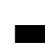 & & $\mathrm{D}$ & & & & 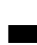 & 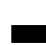 & XXXIV** & $52 / \mathrm{m}$ & & $\mathrm{M}$ & $\mathrm{T}$ & & & & \\
\hline $\mathrm{II}_{+502 \mathrm{~V}} * *$ & $6 / \mathrm{n}$ & & & & $\mathrm{D}$ & & V & & & & $+552 \mathrm{~S}$ & $53 / \mathrm{m}$ & & M & $\mathrm{T}$ & & & & \\
\hline & & & & & & & & & & & $+502 \mathrm{~V}$ & $53 / \mathrm{m}$ & & M & $\mathrm{T}$ & & & & \\
\hline III & $4 / n$ & & & & $\mathrm{D}$ & & V & & $\mathbf{E}$ & & XXXV & $31 / \mathrm{sm}$ & & $\mathrm{M}$ & $\mathrm{T}$ & & & & \\
\hline IV & $5 / \mathrm{n}$ & & & - & $\mathrm{D}$ & & - & S & 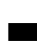 & 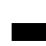 & XXXVI* & $33 / \mathrm{m}$ & & - & - & & & & \\
\hline $\mathrm{V}^{*}$ & $6 / n$ & & & & $\mathrm{D}$ & & & $S$ & 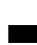 & 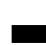 & $37 * *$ & $61 / \mathrm{m}$ & & 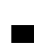 & 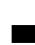 & & & & \\
\hline VI & $4 / n$ & & 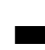 & 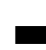 & $\mathrm{D}$ & & - & & 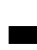 & $\mathrm{L}$ & 38 & $33 / \mathrm{m}$ & $\mathrm{V}$ & $\mathrm{M}$ & $\mathrm{T}$ & & & 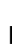 & \\
\hline VII & $5 / \mathrm{n}$ & & 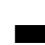 & 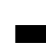 & $\mathrm{D}$ & & V & $\mathrm{S}$ & 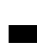 & 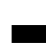 & 39 & $26 / \mathrm{sm}$ & 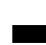 & D & - & D & & & \\
\hline VIII & $4 / \mathrm{n}$ & & 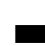 & 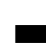 & $\mathrm{D}$ & & V & S & 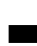 & $\mathrm{L}$ & 40 & $2 / \mathrm{n}$ & & 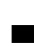 & 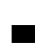 & $\mathrm{D}$ & & & \\
\hline $\mathrm{IX}^{* *}$ & $4 / n$ & & - & - & $\mathrm{D}$ & & - & & 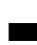 & $\mathrm{L}$ & 41 & $13 / \mathrm{n}$ & & - & - & $\mathrm{D}$ & & & \\
\hline$X$ & $59 / \mathrm{m}$ & & $\mathrm{M}$ & $\mathrm{T}$ & - & & - & 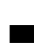 & $\mathrm{S}$ & - & $42 * *$ & $53 / \mathrm{m}$ & 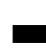 & $\mathrm{M}$ & $\mathrm{T}$ & & & & \\
\hline XI & $6 / n$ & & 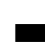 & 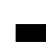 & $\mathrm{D}$ & 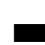 & V & 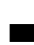 & - & $\mathrm{L}$ & 43 & $5 / \mathrm{n}$ & 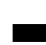 & - & - & D & & & \\
\hline XII* & $5 / \mathrm{n}$ & & & . & D & & - & & 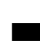 & S & 44 & $6 / n$ & & E & - & $\mathrm{D}$ & & & \\
\hline XIII* & $6 / n$ & & - & & $\mathrm{D}$ & & V & & 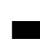 & S & 45 & $10 / \mathrm{n}$ & & — & — & & & & \\
\hline XIV & $5 / \mathrm{n}$ & & & - & $\mathrm{D}$ & & & & 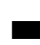 & & 46 & $5 / \mathrm{n}$ & & - & - & $\mathrm{D}$ & & & \\
\hline XV & $1 / \mathrm{n}$ & & E & & 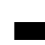 & & - & & & & 47 & $39 / \mathrm{m}$ & & M & $\mathrm{T}$ & & & & \\
\hline XVI & $6 / n$ & & 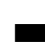 & 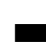 & $\mathrm{D}$ & & $\mathrm{V}$ & & 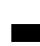 & & 48 & $11 / \mathrm{n}$ & & - & - & $\mathrm{D}$ & & & \\
\hline XVII ** & $9 / \mathrm{n}$ & & - & 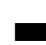 & $\mathrm{D}$ & & V & $S$ & 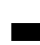 & & 49 & $6 / n$ & & - & - & D & & & \\
\hline XVIII* & $8 / \mathrm{n}$ & & - & - & $\mathrm{D}$ & & $\mathrm{T}$ & $S$ & & & 50 & $5 / \mathrm{n}$ & & - & - & $\mathrm{D}$ & & & \\
\hline XIX & $8 / \mathrm{n}$ & & - & - & D & & 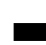 & & & & 51 & $55 / \mathrm{m}$ & & $\mathrm{M}$ & $\mathrm{T}$ & & & & \\
\hline XX & $7 / \mathrm{n}$ & & - & - & D & & - & & 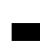 & & 52 & $54 / \mathrm{m}$ & & $\mathrm{M}$ & $\mathrm{T}$ & & & & \\
\hline $\mathrm{XXI}^{* *}$ & $12 / \mathrm{n}$ & & 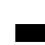 & 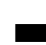 & D & & V & 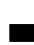 & 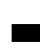 & & 53 & $53 / \mathrm{m}$ & $\mathbf{-}$ & M & $\mathrm{T}$ & & & & \\
\hline XXII & $11 / \mathrm{n}$ & & 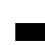 & 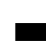 & D & & E & & 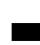 & & 54 & $6 / n$ & 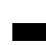 & - & - & $\mathrm{D}$ & & & \\
\hline XXIII & $22 / \mathrm{sm}$ & & 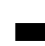 & 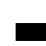 & D & & 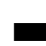 & & E & & 55 & $53 / \mathrm{m}$ & - & $\mathrm{M}$ & $\mathrm{T}$ & & & & \\
\hline XXIV & $9 / \mathrm{n}$ & & - & - & $\mathrm{D}$ & & 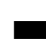 & $\mathrm{S}$ & 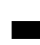 & $\mathrm{S}$ & 56 & $10 / \mathrm{n}$ & & - & - & $\mathrm{D}$ & & & \\
\hline XXV & $58 / \mathrm{m}$ & & M & $\mathrm{T}$ & - & & 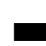 & I & $S$ & & 57 & $7 / \mathrm{n}$ & & - & - & D & & & \\
\hline XXVI & $52 / \mathrm{m}$ & & M & $\mathrm{T}$ & - & & V & & 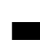 & & 58 & $54 / \mathrm{m}$ & - & $\mathrm{M}$ & $\mathrm{T}$ & & & & \\
\hline XXVII & $58 / \mathrm{m}$ & & M & $\mathrm{T}$ & - & & 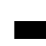 & & S & & 59 & $57 / \mathrm{m}$ & V & $\mathrm{M}$ & $\mathrm{T}$ & & & & \\
\hline XXVIII & $60 / \mathrm{m}$ & & M & $\mathrm{T}$ & I & & 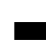 & & S & & $60 * *$ & $49 / \mathrm{m}$ & V & $\mathrm{M}$ & $\mathrm{T}$ & & & & \\
\hline XXIX & $58 / \mathrm{m}$ & & M & $\mathrm{T}$ & 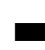 & & 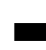 & & $\mathrm{S}$ & & 61 & $6 / n$ & 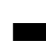 & - & 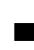 & & & & \\
\hline $\mathrm{XXX}$ & $59 / \mathrm{m}$ & & M & $\mathrm{T}$ & 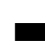 & & V & & $S$ & & 62 & $26 / \mathrm{sm}$ & & - & 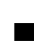 & & & & \\
\hline XXXI & $60 / \mathrm{m}$ & & M & $\mathrm{T}$ & 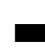 & & - & & S & & 63 & $44 / \mathrm{m}$ & 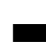 & $\mathrm{M}$ & $\mathrm{T}$ & & & & \\
\hline XXXII & $53 / \mathrm{m}$ & 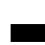 & M & $\mathrm{T}$ & 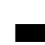 & 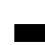 & 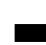 & & $\mathrm{S}$ & $\mathrm{L}$ & $64 * *$ & $57 / \mathrm{m}$ & $\mathrm{V}$ & M & $\mathrm{T}$ & & & & \\
\hline
\end{tabular}

Changes aa: ins346, T484, A502, G543, G546, P552 were named in some former works: ins 345a, T483, A501, G542, G545, P551, respectively (filled square box) insertion-wild type (no change), $N C$ number of changes, $T C$ type of changes, $m$ mosaic, $n$ non-mosaic, $s m$ semi-mosaic, $a a$ amino acids, $A$ alanine, $D$ aspartic acid, $G$ glycine, $I$ isoleucine, $L$ leucine, $M$ methionine, $P$ proline, $S$ serine; $V$ Valine

$*$ Strains with MIC of ceftriaxone $=0.25 \mathrm{mg} / \mathrm{L}$ were described; $* *$ strains with $\mathrm{MIC}$ of ceftriaxone $\geq 0.5 \mathrm{mg} / \mathrm{L}$ were described

substitution) in penicillinase plasmids, TEM-75 (L21F, R164H and T265M substitutions; 2be group-ESBL), TEM141 (K34E substitution; 2b group), TEM-198 (T271I substitution) [67, 70-73]. In addition, mutations causing P14T and P14S amino acid substitutions in the TEM-1 leader peptide, occurring in the Africa and Asia plasmids, have been described, which may be important in the expression of bla genes [71].

Practically, all TEM beta-lactamases described so far in $N$. gonorrhoeae have a narrow substrate spectrum that 
comprises penicillins, characteristic of group $2 b$ beta-lactamases according to Bush classification $[74,75]$. TEM-75 the only ESBL is very rare, and $N$. gonorrhoeae strain containing the plasmid was susceptible to ceftriaxone. The expression of the plasmid bla gene is probably dependent on two factors located in the chromosome: an inducer and a negative regulator of beta-lactamase expression. One may express a concern that the massive use of ESC in many countries for gonorrhoea treatment creates possibility of selecting new variants of TEM beta-lactamase, among which extended spectrum beta-lactamases (ESBLs) belonging to the $2 b$ group, hydrolyzing oxyimino-beta-lactams, including ceftriaxone and cefixime, seem particularly dangerous. This appears particularly likely for a large number of beta-lactamase-producing strains. Although the number of $N$. gonorrhoeae strains producing beta-lactamases is not high in most European countries, the percentage of such strains in some Asian countries, such as Thailand, India and Bhutan is very high and in recent years ranged from 84 to $89 \%$ [76-78].

The highest percentage of $N$. gonorrhoeae producing beta-lactamase in Europe in 2016 was recorded in Sweden (24\%), Austria (23.1\%), Czech Republic (21.1\%), Greece (20\%), Malta (20\%), Poland (18\%) Slovakia (17,3\%), Norway (16,2\%), Belgium (16,2\%) and Spain (16.2\%). In Poland, we observed an increase in the number of beta-lactamase-producing $N$. gonorrhoeae strains from $1.1 \%$ in 2006 to $18 \%$ in $2016[62,63]$.

\section{Resistance to macrolides}

Azithromycin is one of the most commonly used antibiotics from the group of macrolides, also in the treatment of sexually transmitted diseases. The mechanism of azithromycin action is based on the inhibition of bacterial protein synthesis as a result of binding to the $\mathrm{V}$ domain of the $23 \mathrm{~S}$ rRNA, within the 50S subunit of the bacterial ribosome.

$N$. gonorrhoeae azithromycin resistance can have many causes. Typically, the MIC values for azithromycin are determined by several mechanisms occurring simultaneously. Probably, mutations in the gene encoding the $23 \mathrm{~S}$ rRNA V domain are the most common cause of resistance to azithromycin in the currently dominant clones. Mutations can occur in all four gene alleles or in a fewer number of alleles. Two such mutations have been described [79]. The C2611T mutation, whose presence in three or four alleles of the gene, determines the azithromycin MIC at the level of $2-16 \mathrm{mg} / \mathrm{L}$, and the presence of mutations in only one allele determines MIC in the range of $0.06-0.125 \mathrm{mg} / \mathrm{L}$, i.e., such as in strains without mutations. The C2611T mutation, together with the overproduction of MtrCDE pump proteins, is likely the cause of resistance to azithromycin in majority of isolates belonging to the most widespread $N$. gonorrhoeae clone, NG-MAST ST1407, in Europe. The second mutation, A2059T, if it occurs simultaneously in three or four alleles of the gene, determines the high azithromycin MIC values of $>256 \mathrm{mg} / \mathrm{L}$, and the presence of mutations in only one allele does not increase the MIC value [80, 81]. The third type of mutation, A2143G, occurring in all four alleles and conditioning azithromycin MIC of $>256 \mathrm{mg} / \mathrm{L}$, has been described in some strains isolated in England [82].

The second group of mechanisms reducing the susceptibility to macrolides occurring in $N$. gonorrhoeae is the overproduction of MtrCDE or MacAB membrane pump proteins $[83,84]$. The MtrCDE pump removes beta-lactams, macrolides, tetracyclines, rifampicin and detergents. It does not cause resistance alone, but increases the MIC value. The operon encoding the MtrCDE membrane pump is regulated by the MtrR repressor and the MtrA activator. The MtrR protein is involved not only in the regulation of the MtrCDE pump, but also in the regulation of approximately 65 other genes [85]. Overproduction of pump proteins may be caused by mutations in the promoter region of the $m t r R$ gene (e.g., deletion of adenine at the $35 \mathrm{bp}$ position preceding the $\mathrm{mtr} R$ gene), mutations within the $m t r R$ gene and mutations conditioning the formation of a new $m+r C D E$ promoter, the socalled $m$ tr 120 not regulated by MtrR and MtrA [83, 85-87]. These mechanisms, occurring alone, are able to increase azithromycin MIC to $0.5 \mathrm{mg} / \mathrm{L}$, and thus are not able to condition azithromycin resistance. When combined with other resistance mechanisms, they increase the MIC values. The MacAB pump removes only macrolides, and its overproduction is related to $m a c A B$ operon promoter mutations $[84,87]$.

The third group of mechanisms is the synthesis of $23 \mathrm{~S}$ rRNA methylases: ErmB, ErmF, ErmC, ErmA (they cause dimethylation of adenine A2058 within domain $\mathrm{V}$ of $23 \mathrm{~S}$ rRNA) and synthesis of the transferable MefA/E membrane pump. These mechanisms are characteristic of many Grampositive bacteria [88]. Genes conditioning these mechanisms are present in plasmids or transposons and were uptaken by $N$. gonorrhoeae from other bacteria. These mechanisms occurred in $N$. gonorrhoeae with a high frequency in strains isolated in 1940-1987 [28, 86]. They are very rare or nonexistent in the currently isolated strains [24].

Highest percentage of $N$. gonorrhoeae resistant to azithromycin in 2016 (EUCAST breakpoint was MIC $>0.5 \mathrm{mg} / \mathrm{L}$ ) occurred in Portugal (34.5\%), Norway (16.2\%), Hungary (16\%), Greece (14\%), Iceland (14.3\%), Greece (39.6\%), Ireland (37.6\%), Latvia (14.3\%) and France (10.9\%) [61]. Overall, 75 azithromycin-resistant $N$. gonorrhoeae strains isolated in 17 European countries from 2009 to 2014 contained the 23S rRNAA2059G mutation in all four alleles (in four cases MIC $\geq 256 \mathrm{mg} / \mathrm{L}$ ) or C2611T mutation in two to four alleles of the gene (remaining 71 cases) [89]. The frequency of azithromycin and ciprofloxacin resistance in several European countries in 2015-2017 is presented in 
Table 5 Antimicrobial resistance of Neisseria gonorrhoeae in Europe

\begin{tabular}{|c|c|c|}
\hline \multirow[t]{3}{*}{ Country } & \multicolumn{2}{|l|}{$\%$ of resistance* } \\
\hline & \multicolumn{2}{|c|}{ Year: 2014/2015/2016/2017 } \\
\hline & Azithromycin & Ciprofloxacin \\
\hline Austria & $2.0 / 3.3 / 4.7 / 3.8$ & $52.5 / 65.6 / 65.6 / 50.0$ \\
\hline Belgium & 3.6/3.0/9.1/12.2 & $57.9 / 49.5 / 44.4 / 46.9$ \\
\hline Denmark & $3.7 / 2.7 / 1.8 / 12.7$ & $33.0 / 30.9 / 18.9 / 28.0$ \\
\hline France & $10.9 / 5.7 / 7.1 / 6.4$ & $50.9 / 41.9 / 37.4 / 34.5$ \\
\hline Germany & $2.8 / 1.8 / 0.9 / 4.5$ & $63.2 / 61.5 / 58.7 / 61.0$ \\
\hline Greece & $39.6 / 22.0 / 14.0 / 7.9$ & $70.0 / 77.0 / 60.0 / 55.1$ \\
\hline Hungary & $1.1 / 4.7 / 16.0 / 8.1$ & $55.1 / 53.1 / 40.4 / 46.8$ \\
\hline Iceland & $0.0 / 0.0 / 14.3 / 11.6$ & $58.3 / 28.6 / 77.1 / 42.5$ \\
\hline Ireland & $37.6 / 18.2 / 15.0 / 9.8$ & $34.7 / 45.5 / 42.0 / 51.5$ \\
\hline Italy & $6.0 / 2.0 / 11.0 / 16.0$ & $78.0 / 71.0 / 53.0 / 58.0$ \\
\hline Malta & $4.8 / 13.8 / 8.0 / 25.9$ & $57.1 / 65.5 / 44.0 / 63.0$ \\
\hline Netherlands & $1.8 / 4.0 / 2.0 / 5.6$ & $32.2 / 37.0 / 29.4 / 31.0$ \\
\hline Norway & $5.5 / 3.6 / 16.2 / 14.0$ & 73.6/58.7/46.0/43.9 \\
\hline Poland & $8.7 / 5.4 / 2.6 / 12.3$ & $65.2 / 57.1 / 57.1 / 76.9$ \\
\hline Portugal & $17.3 / 17.3 / 34.5 / 12.7$ & $36.4 / 37.3 / 46.4 / 46.4$ \\
\hline Slovakia & $3.7 / 1.9 / 0.9 / 6.4$ & $67.9 / 53.8 / 56.4 / 62.7$ \\
\hline Slovenia & $2.4 / 0.0 / 8.5 / 2.3$ & $45.1 / 34.9 / 33.0 / 42.1$ \\
\hline Spain & $6.6 / 3.0 / 4.1 / 5.5$ & 67.5/65.3/57.5/53.9 \\
\hline Sweden & $4.0 / 14.0 / 5.0 / 3.0$ & $57.0 / 45.0 / 47.0 / 35.0$ \\
\hline United Kingdom & $0.9 / 12.6 / 3.0 / 11.3$ & 33.3/39.7/29.6/34.6 \\
\hline EU/EEA & 7.9/7.1/7.5/7.5 & $50.7 / 49.4 / 46.5 / 46.5$ \\
\hline
\end{tabular}

EU/EEA European Union/European Economic Area

*Azithromycin, breakpoint MIC >0.5 mg/L (EUCAST 2014-2017; ciprofloxacin, breakpoint MIC >0.06 mg/L (EUCAST 2014-2017)

Table 5 [53, 90-92]. Another problem is that azithromycin can be less effective in oropharyngeal than urogenital gonococcal infections [93].

\section{Resistance to fluoroquinolones}

The mechanism of ciprofloxacin action is based on the inactivation of two bacterial enzymes: topoisomerase II (gyrase) and topoisomerase IV, responsible for DNA superspiralization. N.gonorrhoeae ciprofloxacin resistance is most often conditioned by mutations in the $\operatorname{gyrA}$ and $\operatorname{parC}$ genes, coding for topoisomerases II and IV, respectively. Simultaneous combination of changes in both proteins usually determines the resistance. There are several most frequently described substitutions, but they occur at different frequencies depending on the geographical region. The most frequently described substitutions in the $N$. gonorrhoeae GyrA protein include: S91F or T [94-96], A92P, D95N or A or G, I97M and Q102H [94, 96]. The most frequently described substitutions in the $N$. gonorrhoeae ParC protein include: D86N, S87N or I or R, S88P, E91K or G or A and
L106I. Synonymous (silent) mutations that did not change the amino acid were also described in the $\operatorname{parC}$ gene, e.g., codons Y104 (TAT/TAC), A129 (GCG/GCA), L131 (CTC/ CTG) $[94,96] . N$. gonorrhoeae strains that are highly resistant to fluoroquinolones usually have a combination of three or four mutations. The most common combinations of amino acid substitutions in the GyrA and ParC proteins conditioning resistance to fluoroquinolones are: $\mathrm{S} 91 \mathrm{~F}+\mathrm{D} 95 \mathrm{G} / \mathrm{A}$ in GyrA and S87R in ParC. This combination was found in more than $40 \%$ of $N$. gonorrhoeae strains resistant to fluoroquinolones and conditioned ciprofloxacin MIC from 4 to $32 \mathrm{mg} / \mathrm{L}$ [96]. Other common combinations include: $\mathrm{S} 91 \mathrm{~F}+\mathrm{D} 95 \mathrm{~A}$ in GyrA and D86N in ParC (11\% of strains, ciprofloxacin MIC from 4 to $32 \mathrm{mg} / \mathrm{L}$ ) and $\mathrm{S} 91 \mathrm{~F}+\mathrm{D} 95 \mathrm{~N}$ in GyrA and S87N in ParC (10\% of strains, ciprofloxacin MIC from 1 to $2 \mathrm{mg} / \mathrm{L}$ ) [96]. The frequency of individual mutations in the gyrA and $\operatorname{parC}$ genes is varied. Mutations conditioning changes in the GyrA protein in the group of strains isolated in India, in 2007-2009: S91F or T, D95G/N, occurred in all strains, and mutation conditioning the change in the ParC protein: E91G in $46.9 \%$ of strains [95]. Mutations conditioning changes in the GyrA protein in the group of strains isolated in Brazil, in 2006-2010 occurred at the following frequencies: S91F (40\%), D95G (40\%), Q102H (12\%), D95Y (4\%) and mutations conditioning changes in the ParC protein occurred at a frequency of: S87R (40\%), S88T (4\%) [97]. S91F and D95G substitutions of amino acids in the GyrA protein and the S87R substitution in the ParC protein were reported in the most common European clone, ST1407, in ciprofloxacin-resistant strains (MIC $16-32 \mathrm{mg} / \mathrm{L}$ ) [24]. All N. gonorrhoeae strains isolated in England and Wales in 2005-2009 showed high ciprofloxacin resistance (MIC $\geq 16 \mathrm{mg} / \mathrm{L}$ ), 74\% was the ST1407 clone [98].

A mechanism increasing fluoroquinolone MIC values, based on overproduction of NorM membrane pump proteins, was also described in single $N$. gonorrhoeae strains [95-97, 99]. The highest percentage of ciprofloxacin-resistant $N$. gonorrhoeae among European countries in 2016 was recorded in Iceland (77.1\%), Croatia (66.7\%), Austria (65.6\%), Germany (58.7\%) [61].

\section{Resistance to tetracyclines}

Tetracyclines are not currently used in Europe for the treatment of $N$. gonorrhoeae infections as monotherapy, which is due to significant resistance. However, both tetracycline and doxycycline are sometimes used in a combination therapy, with third-generation cephalosporins, alternatively to azithromycin, mainly in mixed infections, e.g., with gonorrhoea and chlamydia coexistence [100-104]. 
The mechanism of antibacterial activity of tetracyclines results from their ability to bind to the $30 \mathrm{~S}$ ribosomal subunit, which leads to protein synthesis inhibition. Resistance in most bacteria is associated with the presence of one of the two basic mechanisms. The first is based on removing the antibiotic from the cell by a variety of membrane pumps, e.g., proteins from TetA to TetL, belonging to the MFS superfamily. The second results from the active protection of the target site on the ribosome $30 \mathrm{~S}$ subunit by proteins named from TetM to TetW [88, 105]. Resistance to tetracyclines in $N$. gonorrhoeae may be conditioned by the conjugative plasmids encoding the TetM protein (active ribosome protection) and then tetracycline MIC is $16-64 \mathrm{mg} / \mathrm{L}$. It was found at the beginning of the 1990s that conjugative plasmids, conditioning tetracycline resistance in $N$. gonorrhoeae, occur in two types, both $25.2 \mathrm{MDa}$ and both contain the tet $M$ gene. Differences were demonstrated on the basis of restriction mapping and southern blot hybridization. One type was named Dutch and the other type American $[106,107]$. Sequencing of the tet $M$ genes conducted in later years also showed the presence of two types, which were named Dutch and American types, respectively, taking the name from the source plasmid type. It is currently known that the plasmid type is usually, but not always, compatible with the type of the tet $M$ determinant [108]. Other mechanisms resulting from mutations in chromosomal genes are responsible for tetracycline resistance in epidemic strains, e.g., pen $B$ encoding the $\mathrm{B}$ porin protein $[25,109]$, pen $C$ encoding PilQ secretin [110], rps $J$ encoding a ribosomal S10 protein $[94,111]$ and overproduction of MtrCDE membrane pump proteins, associated with mutations in promoter regions of $m t r R$ and $m t r C$ genes and the $m t r R$ gene [83, $109,112]$. These mechanisms condition a significantly lower level of resistance, which determines the tetracycline MIC at $2-4 \mathrm{mg} / \mathrm{L}$.

It was demonstrated that the Dutch-type plasmids were more frequent in Asian countries than American ones among $N$. gonorrhoeae strains showing a high level of resistance to tetracyclines, e.g., they represented over $99 \%$ plasmids in China, similarly in Indonesia, Philippines, Thailand [57, $113,114]$ and Bangladesh [115]. The Dutch type was also predominant in the countries of South America, such as Brazil, Guyana or Trinidad [116, 117].

Studies conducted in some European countries, involving strains resistant to tetracyclines, isolated in 1988-1995, showed that American-type plasmids were more common $(81.8 \%)$ than the Dutch ones [114]. The Dutch type was dominant among strains isolated in our center in Warsaw in $2013(88.9 \%)$ [118].

The incidence of tetracycline resistance in $N$. gonorrhoeae depends on the period and country where bacteria were isolated. In China, the incidence of $N$. gonorrhoeae strains with the TetM determinant increased 18-fold in
1999-2005, reaching over 32\% [113]. The frequency of tetracycline resistance in Poland in 2010-2013 was found to remain at a similarly high level (from 40 to over 50\%) during this period $[63,118]$. Similarly, a high percentage of $N$. gonorrhoeae strains resistant to tetracycline was recorded in Germany (over 41\%) [119], Belarus (40\%) [120] and Indonesia, where the percentage of resistant strains was even $100 \%$ in 2010-2012 [76]. Fewer, but also a significant number of tetracycline-resistant strains were found in a similar period in India (12\%) [77], Sri Lanka (16.3\%) [76] and Russia (16.9\%) [94].

\section{Resistance to spectinomycin}

Spectinomycin belongs to the aminocyclitol antibiotics and, like other drugs in this group, binds to the bacterial $30 \mathrm{~S}$ ribosomal subunit, which results in protein synthesis inhibition. Spectinomycin has been used since the early 1960 s, basically exclusively for gonorrhoea treatment. The application of spectinomycin was limited to penicillin-allergic patients or in case of therapeutic failure with penicillin therapy, most often resulting from the production of beta-lactamase by $N$. gonorrhoeae strain. Although $N$. gonorrhoeae strains with a high level of resistance $(\mathrm{MIC}=2048 \mathrm{mg} / \mathrm{L})[121,122]$ were already described in the late 1970s, current studies demonstrate that this resistance is extremely rare or virtually non-existent. Despite the high susceptibility of $N$. gonorrhoeae strains, spectinomycin is not recommended as a first-line drug for gonorrhoea treatment in Europe, because it is less effective in oropharyngeal infections due to poor distribution in the human body [123, 124]. The drug is not only ineffective in other sexually transmitted infections but also could delay incubation period and hinder diagnosis of syphilis. There is also a problem with spectinomycin accessibility in many European countries and in North America. However, due to the low price and considerable effectiveness, it is still used as an alternative medicine in some Asian countries, such as South Korea or in South America [84, 125].

The reported resistance to spectinomycin in $N$. gonorrhoeae is mutational, and mutations affect either 16S rRNA-encoding genes, specifically $r r s 16 \mathrm{~S}$ rRNA (G1064C, C1192U or C1192T) [126] or the rpsE gene encoding the S5 ribosomal protein [127]. Resistant strains, as opposed to, e.g., penicillin-resistant ones, have not spread and are very rare $[128,129]$. For example, the frequency of resistant strains in a study of over 4500 isolates from six Asian countries was found to be $0.17 \%$ [60]. Most often, this type of research does not detect resistant strains $[4,24,120]$. 


\section{Conclusion}

In Europe, gonorrhoea is usually treated with either ceftriaxone monotherapy or, more preferably, ceftriaxone in combination with azithromycin. The recent emergence of $N$. gonorrhoeae strains resistant to ceftriaxone and the threat of their spread and the gradual increase in a proportion of azithromycin-resistant strains may in the future lead to major difficulties in empiric treatment of this disease. There is a risk of spreading of existing ceftriaxone- and cefiximeresistant clones, especially the resistant variants of the clonal CC1 complex (G1407 genogroup). Evolution of TEM beta-lactamases to ESBL is also possible, similarly as in Gram-negative bacilli. However, so far, cefixime resistance among N. gonorrhoeae isolated in Europe in 2009-2017 has remained at around $2 \%$ with no apparent upward trend. In addition, a high percentage of resistance to ciprofloxacin $(46.5 \%)$ and azithromycin (7.5\%) was found in N. gonorrhoeae isolated in Europe in 2017. Introduction of new drugs is possible in the perspective of the next few years. On the other hand, returning to spectinomycin treatment, not used in Europe, seems unlikely due to numerous limitations, especially in the treatment of infections in oropharyngeal location.

\section{Complicate with ethical standards}

Conflict of interest The authors declare no conflict of interest.

\section{References}

1. CDC (2014) Sexually transmitted diseases surveillance 2012. US Department of Health and Human Services, Atlanta

2. de Coul EO, Warning T, Koedijk F (2014) Sexual behaviour and sexually transmitted infections in sexually transmitted infection clinic attendees in the Netherlands, 2007-2011. Int J STD AIDS 25(1):40-51. https://doi.org/10.1177/0956462413491736

3. Majewska A, Mlynarczyk-Bonikowska B, Malejczyk M, Mlynarczyk G, Majewski S (2015) Antiviral medication in sexually transmitted diseases. Part II: HIV. Mini Rev Med Chem 15:93-103

4. Jacobsson S, Boiko I, Golparian D, Blondeel K, Kiarie J, Toskin I et al (2018) WHO laboratory validation of Xpert ${ }^{\circledR} \mathrm{CT} / \mathrm{NG}$ and Xpert $^{\circledR}$ TV on the GeneXpert system verifies high performances. APMIS 126(12):907-912

5. Vyth R, Leval A, Eriksson B, Ericson EL, Marions L, Hergens MP (2016) Gonorrhoea diagnostic and treatment uncertainties: risk factors for culture negative confirmation after positive nucleic acid amplification tests. PloS One 11(5):e0155017. https ://doi.org/10.1371/journal.pone.0155017

6. Newman L, Rowley J, Vander Hoorn S, Wijesooriya NS, Unemo $\mathrm{M}$, Low N et al (2015) Global estimates of the prevalence and incidence of four curable sexually transmitted infections in 2012 based on systematic review and global reporting. PloS One 10:e0143304
7. Chen SC, Yin YP, Dai XQ, Unemo M, Chen XS (2014) Antimicrobial resistance, genetic resistance determinants for ceftriaxone and molecular epidemiology of Neisseria gonorrhoeae isolates in Nanjing, China. J Antimicrob Chemother 69:2959-2965

8. Chen SC, Yin YP, Dai XQ, Unemo M, Chen XS (2016) First nationwide study regarding ceftriaxone resistance and molecular epidemiology of Neisseria gonorrhoeae in China. J Antimicrob Chemother 71:92-99

9. Cole MJ, Spiteri G, Jacobsson S, Woodford N, Tripodo F, Amato-Gauci AJ et al (2017) Overall low extended-spectrum cephalosporin resistance but high azithromycin resistance in Neisseria gonorrhoeae in 24 European countries, 2015. BMC Infect Dis 17:617

10. Unemo M, Del Rio C, Shafer WM (2016) Antimicrobial resistance expressed by Neisseria gonorrhoeae: a major global public health problem in the 21st century. Microbiol Spectr 4:1-32

11. Brooks M (2016) CDC finds first cluster of highly resistant gonorrhea in US. Medscape http://www.medscape.com/viewarticl e/869170. Accessed 25 Aug 2018

12. Unemo M, Ringlander J, Wiggins C, Fredlund H, Jacobsson S, Cole $M$ et al (2015) High in vitro susceptibility to the novel spiropyrimidinetrione AZD0914 among contemporary clinical Neisseria gonorrhoeae isolates in 21 European countries. Antimicrob Agents Chemother 59:5220-5225

13. Jacobsson S, Paukner S, Golparian D, Jensen JS, Unemo M (2017) In Vitro activity of the novel pleuromutilin lefamulin (BC-3781) and effect of efflux pump inactivation on multidrug-resistant and extensively drug-resistant Neisseria gonorrhoeae. Antimicrob Agents Chemother. https://doi.org/10.1128/ AAC.01497-17

14. Jacobsson S, Golparian D, Scangarella-Oman N, Unemo M (2018) In vitro activity of the novel triazaacenaphthylene gepotidacin (GSK2140944) against MDR Neisseria gonorrhoeae. J Antimicrob Chemother 73(8):2072-2077

15. Suay-García B, Pérez-Gracia MT (2018) Future prospects for Neisseria gonorrhoeae treatment. Antibiotics 7:2. https://doi. org/10.3390/antibiotics7020049

16. Jönsson A, Foerster S, Golparian D, Hamasuna R, Jacobsson S, Lindberg $M$ et al (2018) In vitro activity and time-kill curve analysis of sitafloxacin against a global panel of antimicrobialresistant and multidrug-resistant Neisseria gonorrhoeae isolates. APMIS 126(1):29-37. https://doi.org/10.1111/apm.12777

17. Riedel S, Vijayakumar D, Berg G, Kang AD, Smith KP, Kirby JE (2019) Evaluation of apramycin against spectinomycin-resistant and -susceptible strains of Neisseria gonorrhoeae. J Antimicrob Chemother. https://doi.org/10.1093/jac/dkz012

18. Butler MM, Waidyarachchi SL, Connolly KL, Jerse AE, Chai W, Lee RE et al (2018) Aminomethyl spectinomycins as therapeutics for drug-resistant gonorrhea and chlamydia coinfections. Antimicrob Agents Chemother 62:5. https://doi.org/10.1128/ AAC.00325-18

19. Jacobsson S, Mason C, Khan N, Meo P, Unemo M (2019) In vitro activity of the novel oral antimicrobial SMT-571, with a new mechanism of action, against MDR and XDR Neisseria gonorrhoeae: future treatment option for gonorrhoea? J Antimicrob Chemother. https://doi.org/10.1093/jac/dkz060

20. Gulati S, Shaughnessy J, Ram S, Rice PA (2019) Targeting lipooligosaccharide (LOS) for a gonococcal vaccine. Front Immunol 10:321. https://doi.org/10.3389/fimmu.2019.00321

21. Rice PA, Shafer WM, Ram S, Jerse AE (2017) Neisseria gonorrhoeae: drug resistance, mouse models, and vaccine development. Annu Rev Microbiol 71:665-686

22. World Health Organization (2017) Global priority list of antibiotic-resistant bacteria to guide research, discovery, and development of new antibiotics. http://www.who.int/medicines/publi 
cations/global-priority-list-antibiotic-resistant-bacteria/en/ Geneva 2017. Accessed 10 Sept 2018

23. Demczuk W, Sidhu S, Unemo M, Whiley DM, Allen VG, Dillon JR et al (2017) Neisseria gonorrhoeae sequence typing for antimicrobial resistance, a novel antimicrobial resistance multilocus typing scheme for tracking global dissemination of $N$. gonorrhoeae strains. J Clin Microbiol 55:1454-1468

24. Endimiani A, Guilarte YN, Tinguely R, Hirzberger L, Selvini S, Lupo A et al (2014) Characterization of Neisseria gonorrhoeae isolates detected in Switzerland (1998-2012): emergence of multidrug-resistant clones less susceptible to cephalosporins. BMC Infect Dis 14:106

25. Jeverica S, Golparian D, Maticic M, Potocnik M, Mlakar BX, Unemo M (2014) Phenotypic and molecular characterization of Neisseria gonorrhoeae isolates from Slovenia, 2006-12: rise and fall of the multidrug-resistant NG-MAST genogroup 1407 clone? J Antimicrob Chemother 69:1517-1525

26. Lind I (1997) Antimicrobial resistance in Neisseria gonorrhoeae. Clin Infect Dis 24(Suppl 1):S93-S97

27. Miller CP, Bohnhoff M (1945) Studies on the action of penicillin; development of penicillin resistance by gonococcus. Proc Soc Exp Biol Med 60:354-356

28. Roberts MC, Chung WO, Roe D, Xia M, Marquez C, Borthagaray G et al (1999) Erythromycin-resistant Neisseria gonorrhoeae and oral commensal Neisseria spp. carry known rRNA methylase genes. Antimicrob Agents Chemother 43:1367-1372

29. Ohnishi M, Saika T, Hoshina S, Iwasaku K, Nakayama S, Watanabe $\mathrm{H}$ et al (2011) Ceftriaxone-resistant Neisseria gonorrhoeae, Japan. Emerg Infect Dis 17:148-149

30. Unemo M, Golparian D, Nicholas R, Ohnishi M, Gallay A, Sednaoui P (2012) High-level cefixime- and ceftriaxone-resistant $N$. gonorrhoeae in France: novel penA mosaic allele in a successful international clone causes treatment failure. Antimicrob Agents Chemother 56:1273-1280

31. Cole MJ, Ison C, Woodford NJ (2018) Transfer of a gonococcal $\beta$-lactamase plasmid into Neisseria gonorrhoeae belonging to the globally distributed ST1407 lineage. J Antimicrob Chemother 73(9):2576-2577

32. Cole MJ, Quaye N, Jacobsson S, Day M, Fagan E, Ison C et al (2019) Ten years of external quality assessment (EQA) of Neisseria gonorrhoeae antimicrobial susceptibility testing in Europe elucidate high reliability of data. BMC Infect Dis 19:281

33. Clinical and Laboratory Standards Institute (CLSI) (2019) Performance standards for antimicrobial susceptibility testing, CLSI document M100-ED29: 2019. Clinical and Laboratory Standards Institute, Wayne

34. European Committee on Antimicrobial Susceptibility Testing (2019) Breakpoints tables for interpretation of MICs and zones diameters. Version 9.0 http://www.eucast.org. Accessed 25 Mar 2019

35. Hamilton HL, Dillard JP (2006) Natural transformation of Neisseria gonorrhoeae: from DNA donation to homologous recombination. Mol Microbiol 59:376-385

36. Spratt BG (1988) Hybrid penicillin-binding proteins in penicillin resistant strains of Neisseria gonorrhoeae. Nature 332:173-176

37. Faruki H, Kohmescher RN, McKinney WP, Sparling PF (1985) A community-based outbreak of infection with penicillin-resistant Neisseria gonorrhoeae not producing penicillinase (chromosomally mediated resistance). N Engl J Med 313:607-611

38. Ohnishi M, Golparian D, Shimuta K, Saika T, Hoshina S, Iwasaku K et al (2011) Is Neisseria gonorrhoeae initiating a future era of untreatable gonorrhea? Detailed characterization of the first strain with high-level resistance to ceftriaxone. Antimicrob Agents Chemother 55:3538-3545

39. Shimuta K, Unemo M, Nakayama S, Morita-Ishihara T, Dorin M, Kawahata T, Ohnishi M (2013) Antimicrobial resistance and molecular typing of Neisseria gonorrhoeae isolates in Kyoto and Osaka, Japan, 2010 to 2012: intensified surveillance after identification of the first strain (H041) with high-level ceftriaxone resistance. Antimicrob Agents Chemother 57:5225-5232

40. Golparian D, Rose L, Lynam A, Mohamed A, Bercot B, Ohnishi $\mathrm{M}$ et al (2018) Multidrug-resistant Neisseria gonorrhoeae isolate, belonging to the internationally spreading Japanese FC428 clone, with ceftriaxone resistance and intermediate resistance to azithromycin, Ireland, August 2018. Euro Surveill. https://doi. org/10.2807/1560-7917.ES.2018.23.47.1800617

41. Whiley DM, Jennison A, Pearson J, Lahra MM (2018) Genetic characterisation of Neisseria gonorrhoeae resistant to both ceftriaxone and azithromycin. Lancet Infect Dis 18(7):717-718

42. Eyre DW, Sanderson ND, Lord E, Regisford-Reimmer N, Chau K, Barker L et al (2018) Gonorrhoea treatment failure caused by a Neisseria gonorrhoeae strain with combined ceftriaxone and high-level azithromycin resistance, England, February 2018. Euro Surveill. https://doi.org/10.2807/1560-7917. ES.2018.23.27.1800323

43. Poncin T, Fouere S, Braille A, Camelena F, Agsous M, Bebear $\mathrm{C}$ et al (2018) Multidrug-resistant Neisseria gonorrhoeae failing treatment with ceftriaxone and doxycycline in France, November 2017. Euro Surveill. https://doi.org/10.2807/1560-7917. ES.2018.23.21.1800264

44. Terkelsen D, Tolstrup J, Johnsen CH, Lund O, Larsen HK, Worning P et al (2017) Multidrug-resistant Neisseria gonorrhoeae infection with ceftriaxone resistance and intermediate resistance to azithromycin, Denmark, 2017. Euro Surveill. https:// doi.org/10.2807/1560-7917

45. Lefebvre B, Martin I, Demczuk W, Deshaies L, Michaud S, Labbé AC et al (2018) Ceftriaxone-resistant Neisseria gonorrhoeae, Canada, 2017. Emerg Infect Dis. https://doi.org/10.3201/ eid2402.171756

46. Lahra MM, Martin I, Demczuk W, Jennison AV, Lee KI, Nakayama SI et al (2018) Cooperative recognition of internationally disseminated ceftriaxone-resistant Neisseria gonorrhoeae strain. Emerg Infect Dis. https://doi.org/10.3201/eid2404.171873

47. Nakayama S, Shimuta K, Furubayashi K, Kawahata T, Unemo M, Ohnishi M (2016) New ceftriaxone- and multidrug-resistant Neisseria gonorrhoeae strain with a novel mosaic penA gene isolated in Japan. Antimicrob Agents Chemother 60(7):4339-4341

48. Gianecini RA, Golparian D, Zittermann S, Litvik A, Gonzalez S, Oviedo C et al (2019) Genome-based epidemiology and antimicrobial resistance determinants of Neisseria gonorrhoeae isolates with decreased susceptibility and resistance to extendedspectrum cephalosporins in Argentina in 2011-16. J Antimicrob Chemother. https://doi.org/10.1093/jac/dkz054

49. Gianecini R, Oviedo C, Stafforini G, Galarza P (2016) Neisseria gonorrhoeae resistant to ceftriaxone and cefixime, Argentina. Emerg Infect Dis 22(6):1139-1141

50. Lahra MM, Ryder N, Whiley DM (2014) A new multidrugresistant strain of Neisseria gonorrhoeae in Australia. N Engl J Med 371:1850-1851

51. Cámara J, Serra J, Ayats J, Bastida T, Carnicer-Pont D, Andreu A et al (2012) Molecular characterization of two high-level ceftriaxone-resistant Neisseria gonorrhoeae isolates detected in Catalonia, Spain. J Antimicrob Chemother 67:1858-1860

52. European Centre for Disease Prevention and Control (2018) Extensively drug-resistant (XDR) Neisseria gonorrhoeae in the United Kingdom and Australia-7 May 2018. ECDC, Stockholm

53. Mlynarczyk-Bonikowska B, Malejczyk M, Majewski S, Unemo M (2018) Antibiotic resistance and NG-MAST sequence types of Neisseria gonorrhoeae isolates in Poland compared to internationally. Post Dermatol Alergol 35(6):546-551

54. Igawa G, Yamagishi Y, Lee KI, Dorin M, Shimuta K, Suematsu $\mathrm{H}$ et al (2018) Neisseria cinerea with high ceftriaxone MIC is 
a source of ceftriaxone and cefixime resistance-mediating penA sequences in Neisseria gonorrhoeae. Antimicrob Agents Chemother. https://doi.org/10.1128/AAC.02069-17

55. Gose S, Nguyen D, Lowenberg D, Samuel M, Bauer H, Pandori M (2013) Neisseria gonorrhoeae and extended-spectrum cephalosporins in California: surveillance and molecular detection of mosaic penA. BMC Infect Dis 13:570

56. Whiley DM, Goire N, Lambert SB, Ray S, Limnios EA, Nissen MD et al (2010) Reduced susceptibility to ceftriaxone in Neisseria gonorrhoeae is associated with mutations G542S, P551S and $\mathrm{P} 551 \mathrm{~L}$ in the gonococcal penicillin-binding protein 2. J Antimicrob Chemother 65:1615-1618

57. Li S, Su X, Le W, Jiang F, Wang B, Rice PA (2014) Antimicrobial susceptibility of Neisseria gonorrhoeae isolates from symptomatic men attending the Nanjing sexually transmitted diseases clinic (2011-2012): genetic characteristics of isolates with reduced sensitivity to ceftriaxone. BMC Infect Dis 14:622

58. Unemo M, Golparian D, Sanchez-Buso L, Grad Y, Jacobsson S, Ohnishi M et al (2016) The novel 2016 WHO Neisseria gonorrhoeae reference strains for global quality assurance of laboratory investigations: phenotypic, genetic and reference genome characterization. J Antimicrob Chemother 71:3096-3108

59. Zhao S, Tobiason DM, Hu M, Seifert HS, Nicholas RA (2005) The penC mutation conferring antibiotic resistance in Neisseria gonorrhoeae arises from a mutation in the PilQ secretin that interferes with multimer stability. Mol Microbiol 57:1238-1251

60. Lee H, Unemo M, Kim HJ, Seo Y, Lee K, Chong Y (2015) Emergence of decreased susceptibility and resistance to extendedspectrum cephalosporins in Neisseria gonorrhoeae in Korea. $\mathrm{J}$ Antimicrob Chemother 70:2536-2542

61. ECDC surveillance report gonococcal antimicrobial susceptibility surveillance in Europe 2016 (2018) European Centre for Disease Prevention and Control, Stockholm htttp://www.ecdc. europa.eu. Accessed 11 Sept 2018

62. Mlynarczyk-Bonikowska B, Kujawa M, Mlynarczyk G, Malejczyk M, Majewski S (2016) Susceptibility to ceftriaxone and penicillinase plasmids of Neisseria gonorrhoeae strains isolated in Poland in 2012-2013. Folia Microbiol 61:269-273

63. Mlynarczyk-Bonikowska B, Serwin AB, Golparian D, Walter de Walthoffen S, Majewski S, Koper M et al (2014) Epidemiology, antimicrobial susceptibility/resistance and genetic characteristics of Neisseria gonorrhoeae isolates from Poland, 2010-2012. BMC Infect Dis 14:65

64. Phillips I (1976) Beta-lactamase-producing, penicillin-resistant gonococcus. Lancet 2:656-657

65. Dillon JAR, Li H, Yeung K, Aman TA (1999) A PCR assay for discriminating Neisseria gonorrhoeae beta-lactamase-producing plasmids. Mol Cell Probes 13:89-92

66. Fayemiwo SA, Muller EE, Gumede L, Lewis DA (2011) Plasmid-mediated penicillin and tetracycline resistance among Neisseria gonorrhoeae isolates in South Africa: prevalence, detection and typing using a novel molecular assay. Sex Transm Dis 38:329-333

67. Ohnishi M, Ono E, Shimuta K, Watanabe H, Okamura N (2010) Identification of TEM-135 beta-lactamase in penicillinaseproducing Neisseria gonorrhoeae strains in Japan. Antimicrob Agents Chemother 54:3021-3023

68. Pagotto F, Aman AT, Ng LK, Yeung KH, Brett M, Dillon JA (2000) Sequence analysis of the family of penicillinase-producing plasmids of Neisseria gonorrhoeae. Plasmid 43:24-34

69. Trembizki E, Buckley C, Lawrence A, Lahra M, Whiley D, GRAND Study Investigators (2014) Characterization of a novel Neisseria gonorrhoeae penicillinase-producing plasmid isolated in Australia in 2012. Antimicrob Agents Chemother 58:4984-4985
70. Gianecini R, Oviedo C, Littvik A, Mendez E, Piccoli L, Montibello $S$ et al (2015) Identification of TEM-135 $\beta$-lactamase in Neisseria gonorrhoeae strains carrying African and Toronto plasmids in Argentina. Antimicrob Agents Chemother 59:717-720

71. Muhammad I, Golparian D, Dillon JA, Johansson A, Ohnishi $\mathrm{M}$, Sethi S et al (2014) Characterization of blaTEM genes and types of $\beta$-lactamase plasmids in Neisseria gonorrhoeae - the prevalent and conserved blaTEM-135 has not recently evolved and existed in the Toronto plasmid from the origin. BMC Infect Dis $14: 454$

72. Micaëlo M, Goubard A, La Ruche G, Denamur E, Tenaillon O, Cambau E et al (2017) Molecular epidemiology of penicillinaseproducing Neisseria gonorrhoeae isolates in France. Clin Microbiol Infect 23:968-973

73. Ryan L, Golparian D, Fennelly N, Rose L, Walsh P, Lawlor B et al (2018) Antimicrobial resistance and molecular epidemiology using whole-genome sequencing of Neisseria gonorrhoeae in Ireland, 2014-2016: focus on extended-spectrum cephalosporins and azithromycin. Eur J Clin Microbiol Infect Dis 37(9):1661-1672. https://doi.org/10.1007/s10096-018-3296-5

74. Bush K, Jacoby GA (2010) Updated functional classification of beta-lactamases. Antimicrob Agents Chemother 54:969-976

75. Bush K, Palzkill T, Jacoby G (2018) Beta-lactamase classification and amino acid sequences for TEM, SHV and OXA extended-spectrum and inhibitor resistant enzymes, Lahey Clinic http://www.lahey.org/Studies/. Accessed 5 Sept 2018

76. Bala M, Kakran M, Singh V, Sood S, Ramesh V, Members of WHO GASP SEAR Network (2013) Monitoring antimicrobial resistance in Neisseria gonorrhoeae in selected countries of the WHO south-east Asia region between 2009 and 2012: a retrospective analysis. Sex Transm Infect 89:iv28-iv35

77. Bharara T, Bhalla P, Rawat D, Garg VK, Sardana K, Chakravarti A (2015) Rising trend of antimicrobial resistance among Neisseria gonorrhoeae isolates and the emergence of $N$. gonorrhoeae isolate with decreased susceptibility to ceftriaxone. Indian J Med Microbiol 33:39-42

78. Lawung R, Cherdtrakulkiat R, Charoenwatanachokchai A, Nabu S, Lokpichart S, Prachayasittikul V (2012) Antimicrobial resistance markers as a monitoring index of gonorrhoea in Thailand. Acta Microbiol Immunol Hung 59:157-169

79. Demczuk W, Martin I, Peterson S, Bharat A, Van Domselaar G, Graham M et al (2016) Genomic epidemiology and molecular resistance mechanisms of azithromycin resistant Neisseria gonorrhoeae in Canada from 1997 to 2014. J Clin Microbiol 54:1304-1313

80. Takayama Y, Nakayama S, Shimuta K, Morita-Ishihara T, Ohnishi M (2014) Characterization of azithromycin-resistant Neisseria gonorrhoeae isolated in Tokyo in 2005-2011. J Infect Chemother 20:339-341

81. Unemo M, Golparian D, Hellmark B (2014) First three Neisseria gonorrhoeae isolates with high-level resistance to azithromycin in Sweden: a threat to currently available dual-antimicrobial regimens for treatment of gonorrhea? Antimicrob Agents Chemother 58:624-625

82. Chisholm SA, Wilson J, Alexander S, Tripodo F, Al-Shahib A, Schaefer U et al (2016) An outbreak of high-level azithromycin resistant Neisseria gonorrhoeae in England. Sex Transm Infect 92:365-367

83. Ohneck EA, Zalucki YM, Johnson PJ, Dhulipala V, Golparian D, Unemo $M$ et al (2011) A novel mechanism of high-level, broad-spectrum antibiotic resistance caused by a single base pair change in Neisseria gonorrhoeae. Mbio 2:e00187-e00211

84. Shams-ur-Rehman Khan A, Amanullah Akhter K (2009) Clinical efficacy of the various drugs used in the treatment of gonorrhoeae. J Ayub Med Coll Abbottabad 21:28-30 
85. Ohneck EA, Goytia M, Rouquette-Loughlin CE, Joseph SJ, Read TD, Jerse AE et al (2015) Overproduction of the MtrCDE efflux pump in Neisseria gonorrhoeae produces unexpected changes in cellular transcription patterns. Antimicrob Agents Chemother 59:724-726

86. Cousin S Jr, Whittington WL, Roberts MC (2003) Acquired macrolide resistance genes in pathogenic Neisseria spp. isolated between 1940 and 1987. Antimicrob Agents Chemother 47:3877-3880

87. Golparian D, Shafer WM, Ohnishi M, Unemo M (2014) Importance of multidrug efflux pumps in the antimicrobial resistance property of clinical multidrug-resistant isolates of Neisseria gonorrhoeae. Antimicrob Agents Chemother 58:3556-3559

88. Mlynarczyk B, Mlynarczyk A, Kmera-Muszyńska M, Majewski S, Mlynarczyk G (2010) Mechanisms of resistance to antimicrobial drugs in pathogenic Gram-positive cocci. Mini Rev Med Chem 10:928-937

89. Jacobsson S, Golparian D, Cole M, Spiteri G, Martin I, Bergheim $\mathrm{T}$ et al (2016) WGS analysis and molecular resistance mechanisms of azithromycin-resistant (MIC $>2 \mathrm{mg} / \mathrm{L}$ ) Neisseria gonorrhoeae isolates in Europe from 2009 to 2014. J Antimicrob Chemother 71:3109-3116

90. European Centre for Disease Prevention and Control (2019) Gonococcal antimicrobial susceptibility surveillance in Europeresults summary 2017. ECDC, Stockholm

91. European Centre for Disease Prevention and Control (2018) Gonococcal antimicrobial susceptibility surveillance in Europe, 2016. ECDC, Stockholm

92. Day MJ, Spiteri G, Jacobsson S, Woodford N, Amato-Gauci AJ, Cole MJ et al (2018) Stably high azithromycin resistance and decreasing ceftriaxone susceptibility in Neisseria gonorrhoeae in 25 European countries, 2016. BMC Infect Dis 18(1):609. https ://doi.org/10.1186/s12879-018-3528-4

93. Kong FYS, Horner P, Unemo M, Hocking JS (2019) Pharmacokinetic considerations regarding the treatment of bacterial sexually transmitted infections with azithromycin: a review. J Antimicrob Chemother. https://doi.org/10.1093/jac/dky548

94. Kubanov A, Vorobyev D, Chestkov A, Leinsoo A, Shaskolskiy B, Dementieva EV et al (2016) Molecular epidemiology of drugresistant Neisseria gonorrhoeae in Russia (current status, 2015). BMC Infect Dis 16:389

95. Kulkarni S, Bala M, Sane S, Pandey S, Bhattacharya J, Risbud A (2012) Mutations in the gyrA and parC genes of quinoloneresistant Neisseria gonorrhoeae isolates in India. Int J Antimicrob Agents 40:549-553

96. Zhao LH, Zhao SP (2013) Molecular basis of high-level ciprofloxacin resistance in Neisseria gonorrhoeae strains from Shandong province, China. Braz J Microbiol 44:273-276

97. Uehara AA, Amorin EL, MdeF Ferreira, Andrade CF, Clementino MB, de Filippis I et al (2011) Molecular characterization of quinolone-resistant Neisseria gonorrhoeae isolates from Brazil. J Clin Microbiol 49:4208-4212

98. Chisholm SA, Alexander S, Desouza-Thomas L, MaclureWebster E, Anderson J, Nichols T et al (2011) Emergence of a Neisseria gonorrhoeae clone showing decreased susceptibility to cefixime in England and Wales. J Antimicrob Chemother 66:2509-2512

99. Lindback E, Rahman M, Jalal S, Wretlind B (2002) Mutations in $\operatorname{gyr} A, \operatorname{gyr} B, \operatorname{parC}$, and parE in quinolone-resistant strains of Neisseria gonorrhoeae. APMIS 110:651-657

100. Barbee LA, Soge OO, Holmes KK, Golden MR (2014) In vitro synergy testing of novel antimicrobial combination therapies against Neisseria gonorrhoeae. J Antimicrob Chemother 69:1572-1578
101. Kerani RP, Stenger MR, Weinstock H, Bernstein KT, Reed M, Schumacher C et al (2015) Gonorrhea treatment practices in the STD surveillance network, 2010-2012. Sex Transm Dis 42:6-12

102. Lechtenberg RJ, Samuel MC, Bernstein KT, Lahiff M, Olson N, Bauer HM (2014) Variation in adherence to the treatment guidelines for Neisseria gonorrhoeae by clinical practice setting, California, 2009 to 2011. Sex Transm Dis 41:338-344

103. Singh AE, Gratrix J, Martin I, Friedman DS, Hoang L, Lester R et al (2015) Gonorrhea treatment failures with oral and injectable expanded spectrum cephalosporin monotherapy vs dual therapy at 4 Canadian sexually transmitted infection clinics, 2010-2013. Sex Transm Dis 42:331-336

104. Starnino S, Neri A, Stefanelli P, Neisseria gonorrhoeae Italian Study Group (2008) Molecular analysis of tetracycline-resistant gonococci: rapid detection of resistant genotypes using a realtime PCR assay. FEMS Microbiol Lett 286:16-23

105. Roberts MC (2011) Environmental macrolide-lincosamidestreptogramin and tetracycline resistant bacteria. Front Microbiol 2:1-8

106. Chalkley LJ, Janse van Rensburg MN, Matthee PC, Ison CA, Botha PL (1997) Plasmid analysis of Neisseria gonorrhoeae isolates and dissemination of tet $M$ genes in southern Africa 1993-1995. J Antimicrob Chemother 40:817-822

107. Gascoyne DM, Heritage J, Hawkey PM, Turner A, van Klingeren B (1991) Molecular evolution of tetracycline-resistance plasmids carrying TetM found in Neisseria gonorrhoeae from different countries. J Antimicrob Chemother 28:173-183

108. Pachulec E, Van der Does C (2010) Conjugative plasmids of Neisseria gonorrhoeae. PLoS One 5:e9962

109. Thakur SD, Starnino S, Horsman GB, Levett PN, Dillon JR (2014) Unique combined penA/mtrR/porB mutations and NGMAST strain types associated with ceftriaxone and cefixime MIC increases in a 'susceptible' Neisseria gonorrhoeae population. $\mathrm{J}$ Antimicrob Chemother 69:1510-1516

110. Whiley DM, Jacobsson S, Tapsall JW, Nissen MD, Sloots TP, Unemo M (2010) Alterations of the pilQ gene in Neisseria gonorrhoeae are unlikely contributors to decreased susceptibility to ceftriaxone and cefixime in clinical gonococcal strains. J Antimicrob Chemother 65:2543-2547

111. Hu M, Nandi S, Davies C, Nicholas RA (2005) High-level chromosomally mediated tetracycline resistance in Neisseria gonorrhoeae results from a point mutation in the rpsJ gene encoding ribosomal protein $\mathrm{S} 10$ in combination with the $m t r R$ and penB resistance determinants. Antimicrob Agents Chemother 49:4327-4334

112. Hess D, Wu A, Golparian D, Esmaili S, Pandori W, Sena E et al (2012) Genome sequencing of a Neisseria gonorrhoeae isolate of a successful international clone with decreased susceptibility and resistance to extended-spectrum cephalosporins. Antimicrob Agents Chemother 56:5633-5641

113. Su X, Jiang F, Qimuge Dai X, Sun H, Ye S (2007) Surveillance of antimicrobial susceptibilities in Neisseria gonorrhoeae in Nanjing, China, 1999-2006. Sex Transm Dis 34:995-999

114. Turner A, Gough KR, Leeming JP (1999) Molecular epidemiology of tetM genes in Neisseria gonorrhoeae. Sex Transm Infect 75:60-66

115. Alam MA, Chowdhury ZU, Chowdhury AH, Rahman M (2012) Epidemic plasmids in Neisseria gonorrhoeae isolated from highrisk population in Bangladesh. Mymensingh Med J 21:220-225

116. Dillon JA, Li H, Sealy J, Ruben M, Prabhakar P, Caribbean GASP Network Gonococcal Antimicrobial Surveillance Program (2001) Antimicrobial susceptibility of Neisseria gonorrhoeae isolates from three Caribbean countries: Trinidad, Guyana, and St. Vincent. Sex Transm Dis 28:508-514

117. Dillon JA, Rubabaza JP, Benzaken AS, Sardinha JC, Li H, Bandeira MG et al (2001) Reduced susceptibility to azithromycin 
and high percentages of penicillin and tetracycline resistance in Neisseria gonorrhoeae isolates from Manaus, Brazil, 1998. Sex Transm Dis 28:521-526

118. Mlynarczyk-Bonikowska B, Kujawa M, Malejczyk M, Majewski S, Mlynarczyk G (2016) Plasmid-mediated resistance to tetracyclines among Neisseria gonorrhoeae strains isolated in Poland in the period 2012-2013. Post Dermatol Alergol 33:475-479

119. Horn NN, Kresken M, Körber-Irrgang B, Göttig S, Wichelhaus C, Wichelhaus TA et al (2014) Antimicrobial susceptibility and molecular epidemiology of Neisseria gonorrhoeae in Germany. Int J Med Microbiol 304:586-591

120. Lebedzeu F, Golparian D, Titov L, Pankratava N, Glazkova S, Shimanskaya I, Charniakova N, Lukyanau A, Domeika M, Unemo M (2015) Antimicrobial susceptibility/resistance and NG-MAST characterization of Neisseria gonorrhoeae in Belarus, eastern Europe, 2010-2013. BMC Infect Dis 15:29

121. Ashford WA, Potts DW, Adams HJ, English JC, Johnson SR, Biddle JW et al (1981) Spectinomycin-resistant penicillinase producing Neisseria gonorrhoeae. Lancet 2:1035-1037

122. Ison CA, Littleton K, Shannon KP (1983) Spectinomycin resistant gonococci. Br Med J 287:1827-1829

123. Easmon CS, Forster GE, Walker GD, Ison CA, Harris JR, Munday PE (1984) Spectinomycin as initial treatment for gonorrhoea. Br Med J 289:1032-1034

124. Judson FN, Ehret JM, Handsfield HH (1985) Comparative study of ceftriaxone and spectinomycin for treatment of pharyngeal and anorectal gonorrhea. JAMA 253:1417-1419

125. Starnino S, GASP-LAC Working Group, Galarza P, Carvallo ME, Benzaken AS, Ballesteros AM et al (2012) Retrospective analysis of antimicrobial susceptibility trends (2000-2009) in Neisseria gonorrhoeae isolates from countries in Latin America and the Caribbean shows evolving resistance to ciprofloxacin, azithromycin and decreased susceptibility to ceftriaxone. Sex Transm Dis 39:813-821

126. Ilina EN, Malakhova MV, Bodoev IN, Oparina NY, Filimonova AV, Govorun VM (2013) Mutation in ribosomal protein S5 leads to spectinomycin resistance in Neisseria gonorrhoeae. Front Microbiol 4:186

127. Unemo M, Golparian D, Skogen V, Olsen AO, Moi H, Syversen $\mathrm{G}$ et al (2013) Neisseria gonorrhoeae strain with high-level resistance to spectinomycin due to a novel resistance mechanism (mutated ribosomal protein S5) verified in Norway. Antimicrob Agents Chemother 57:1057-1061

128. Nabu $\mathrm{S}$, Lawung $\mathrm{R}$, Isarankura-Na-Ayudhya $\mathrm{P}$, Isarankura-NaAyudhya C, Roytrakul S, Prachayasittikul V (2014) Reference map and comparative proteomic analysis of Neisseria gonorrhoeae displaying high resistance against spectinomycin. J Med Microbiol 63:371-385

129. Unemo M, Shafer WM (2014) Antimicrobial resistance in Neisseria gonorrhoeae in the 21 st century: past, evolution, and future. Clin Microbiol Rev 27:587-613

Publisher's Note Springer Nature remains neutral with regard to jurisdictional claims in published maps and institutional affiliations. 